\title{
Ultra-high frequency, high Q/volume micromechanical resonators in a planar AIN phononic crystal
}

M. Ghasemi Baboly, S. Alaie, C. M. Reinke, I. El-Kady, and Z. C. Leseman'

Citation: J. Appl. Phys. 120, 034502 (2016); doi: 10.1063/1.4958671

View online: http://dx.doi.org/10.1063/1.4958671

View Table of Contents: http://aip.scitation.org/toc/jap/120/3

Published by the American Institute of Physics

\section{Articles you may be interested in}

Demonstration of acoustic waveguiding and tight bending in phononic crystals

J. Appl. Phys. 109, 183504183504 (2016); 10.1063/1.4966463

\section{A Journal of Applied Physics}

INTRODUCING INVITED PERSPECTIVES Ultrafast magnetism and THz spintronics Authors: Jakob Walowski and Markus Münzenberg 


\title{
Ultra-high frequency, high Q/volume micromechanical resonators in a planar AIN phononic crystal
}

\author{
M. Ghasemi Baboly, ${ }^{1}$ S. Alaie, ${ }^{2,3}$ C. M. Reinke,${ }^{4}$ I. El-Kady ${ }^{4}$ and Z. C. Leseman ${ }^{1, a)}$ \\ ${ }^{1}$ Department of Mechanical and Nuclear Engineering, Kansas State University, Manhattan, Kansas 66506, \\ USA \\ ${ }^{2}$ Dalio Institute of Cardio vascular Imaging, Weill Cornell Medicine and NewYork-Presbyterian Hospital, \\ New York 10021, USA \\ ${ }^{3}$ Department of Radiology, Weill Cornell Medicine, New York 10021, USA \\ ${ }^{4}$ Department of Applied Photonic Microsystems, Sandia National Laboratories, Albuquerque, \\ New Mexico 87175, USA
}

(Received 10 May 2016; accepted 29 June 2016; published online 19 July 2016)

\begin{abstract}
This paper presents the first design and experimental demonstration of an ultrahigh frequency complete phononic crystal (PnC) bandgap aluminum nitride (AIN)/air structure operating in the $\mathrm{GHz}$ range. A complete phononic bandgap of this design is used to efficiently and simultaneously confine elastic vibrations in a resonator. The PnC structure is fabricated by etching a square array of air holes in an AlN slab. The fabricated $\mathrm{PnC}$ resonator resonates at $1.117 \mathrm{GHz}$, which corresponds to an out-of-plane mode. The measured bandgap and resonance frequencies are in very good agreement with the eigen-frequency and frequency-domain finite element analyses. As a result, a quality factor/volume of $7.6 \times 10^{17} / \mathrm{m}^{3}$ for the confined resonance mode was obtained that is the largest value reported for this type of $\mathrm{PnC}$ resonator to date. These results are an important step forward in achieving possible applications of PnCs for RF communication and signal processing with smaller dimensions. Published by AIP Publishing. [http://dx.doi.org/10.1063/1.4958671]
\end{abstract}

\section{INTRODUCTION}

During the last decade, the propagation of elastic waves in periodic or random composite structures has garnered much attention. ${ }^{1,2}$ Phononic crystals (PnCs) are structures that have mechanical properties that vary periodically. ${ }^{3}$ One of their unique abilities is to exhibit a wide acoustic bandgap, which is a range of frequencies for which waves do not propagate through the structure. ${ }^{4}$ Previous studies have shown the versatility and tunability of frequency band structures in PnCs by manipulating the geometry and introducing defects. This great capability allows for confinement, wave guiding, and focusing of mechanical energy. ${ }^{5,6}$ Because of their low loss and high quality characteristics, these devices have applications in wireless communications, opto-mechanical coupling devices, modulators, and related areas.

Recently, numerical and experimental studies on 2D PnC slabs with finite thicknesses were performed, and large acoustic bandgaps have been demonstrated in PnCs made of $\mathrm{Si}, \mathrm{SiO}_{2}, \mathrm{SiN}_{\mathrm{x}}$, and $\mathrm{SiC}$ as the matrix material and $\mathrm{W}$ or air as the inclusion. ${ }^{7-10}$ Different types of lattices have been used, such as square and hexagonal with circular inclusions. ${ }^{11,12}$ In addition, to achieve wider bandgaps, recently some other designs were proposed and tested, including fractal, snowflake, inverse acoustic band gap (IABG), and hybrid. ${ }^{12-15}$

Applications of PnCs combined with microelectromechanical systems (MEMS) interdigitated transducers (IDT) and microresonators to engineer different types of filters or acoustic sensors have attracted much interest recently. Using PnCs, high

\footnotetext{
a) Author to whom correspondence should be addressed. Electronic mail: zleseman@ksu.edu
}

quality factor acoustic cavities or waveguides can be fabricated with very small volume. For this purpose, the transducers used to sense and launch the waves are usually made of piezoelectric materials such as AlN and $\mathrm{ZnO}$. These piezoelectric materials have recently also been used as the host material ${ }^{16,17}$ to reduce the insertion loss from the electrodes to the matrix and attain better confinement. As a result, using a design of PnCs with AIN as the host material, MEMS devices operating at the frequencies of $800,{ }^{18} 586,{ }^{19} 660,{ }^{20}$ and $780 \mathrm{MHz}^{21}$ have been realized.

In this work, ultra-high frequency PnC slabs and microcavities are designed, analyzed, and tested. The PnC used to create the micro-mechanical resonator is made by etching a square array of cross-type holes in a thin slab of AIN. The existence of a bandgap in the $\mathrm{PnC}$ slab and high frequency mode in the resonator is demonstrated at the frequency of $1.117 \mathrm{GHz}$. The realization of the resonator at $\mathrm{GHz}$ frequencies is aligned with previous efforts to prove the performance of PnCs in a new class of microresonators applicable to RF communications.

\section{FINITE ELEMENT ANALYSIS}

The PnC structure designed is formed by embedding a simple cubic array of cross-type holes in a thin AlN matrix, as shown in Fig. 1. This figure shows a unit cell of the PnC structure in which $d$ is the thickness of the PnC structure, $a$ is the lattice constant, and $w$ and $l$ are the width and length of each cross-hole, respectively. For this PnC structure, the geometrical parameters are $a=3.4 \mu \mathrm{m}, d=1.5 \mu \mathrm{m}, w=800 \mathrm{~nm}$, and $l=2.61 \mu \mathrm{m}$. 


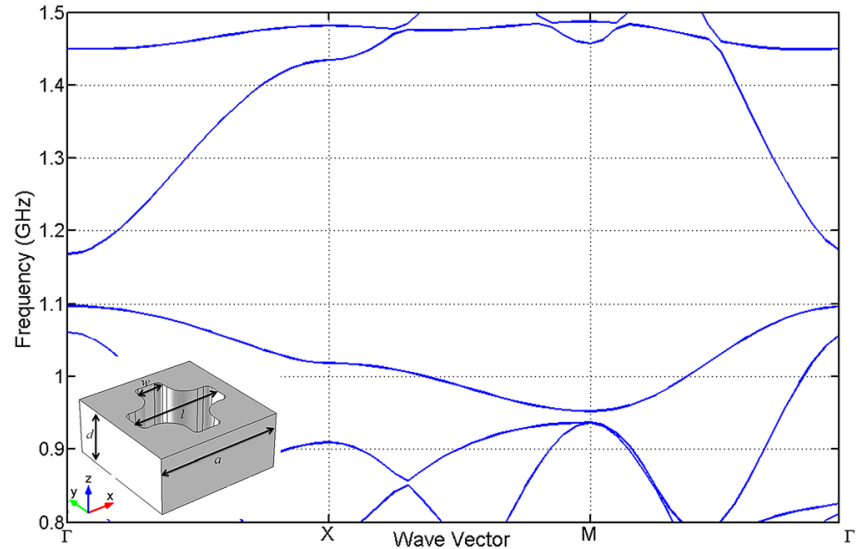

FIG. 1. The band structure of a simple cubic lattice PnC of cross type holes in AlN slab with lattice constant $a=3.41 \mu \mathrm{m}$, width and length of each rectangular hole $w=800 \mathrm{~nm}$ and $l=2.6 \mu \mathrm{m}$, and the slab thickness $d=1.5 \mu \mathrm{m}$. A unit cell of the structure is shown in the inset. (inset) Schematic of the designed cavity that is made in the PnC structure by removing two rows of holes from the PnC structure.

To determine the frequency range over which the bandgap occurs, a three-dimensional COMSOL Multiphysics ${ }^{22}$ model was used. In the FE model, Bloch periodic boundary conditions on the unit cell were applied to simulate an infinite by infinite structure in order to calculate the dispersion relation. The structure was simulated by applying a stress-free boundary condition in the $z$ directions. Then, the eigenfrequencies of the structure were found by solving the elastic wave equation that is described by the Bloch-Floquet Theorem. ${ }^{23}$ In the simulation, all possible modes were considered, i.e., inplane, out-of-plane transverse, and longitudinal modes. Furthermore, the frequency bandgap is determined by sweeping the eigenfrequency solver over the symmetric directions of the irreducible Brillouin zone $(\Gamma-\mathrm{X}-\mathrm{M}-\Gamma)$ for the reciprocal lattice to generate the band diagram shown in Fig. 1.

It is clear that this geometry provides a bandgap in the frequency range of $1095 \mathrm{MHz}<f<1170 \mathrm{MHz}$, which corresponds to $6.6 \%$ gap-to-midgap ratio and allows for the design of other PnC devices to confine mechanical energy.

\section{FABRICATION AND EXPERIMENTATION}

Similar to most MEMS devices, the fabrication process for a PnC consists of a combination of deposition and etching steps followed by a device release step. The PnCs and the IDTs were fabricated in a 7-mask CMOS-compatible process. The entire process is summarized in Fig. 2.

High-resistivity $(>10 \mathrm{k} \Omega \mathrm{cm})$ silicon wafers are selected as the substrate material. A $600 \mathrm{~nm}$-thick oxide deposition is followed by the deposition of a $2 \mu \mathrm{m}$ undoped polysilicon release layer. Patterning and subsequent etching of the polysilicon layer are performed to the level of the deposited silicon dioxide layer. Next, a $3 \mu \mathrm{m}$ PETEOS (oxide) layer is deposited, followed by chemical-mechanical polish (CMP) processing, to the level of the polysilicon layer (Fig. 2(c)).

After patterning and etching of $600 \mathrm{~nm}$ of the oxide, chemical vapor deposition (CVD) is used to make a $1.2 \mu \mathrm{m}$

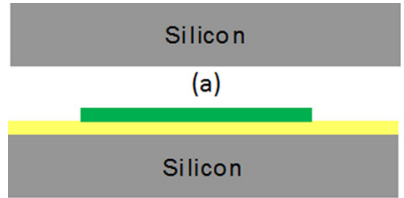

(b)

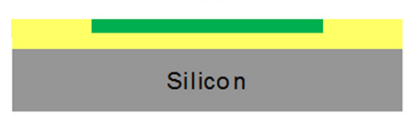

(c)

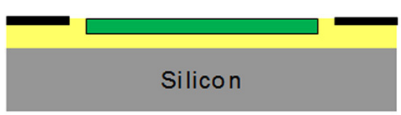

(d)

Silicon $\mathrm{SiO}_{2}$

Polysilicon $\square \mathrm{Al}$

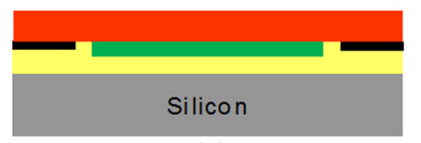

(e)

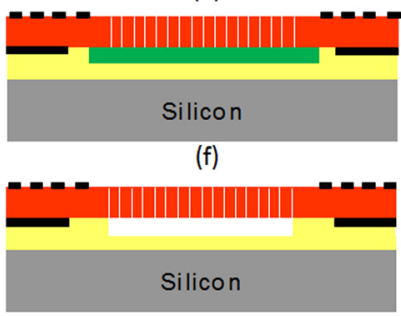

(g)

Aluminum Nitride
FIG. 2. A polysilicon layer is deposited on the oxide which was previously deposited on a silicon substrate. Deposition of a $100 \mathrm{~nm}$ bottom layer oxide is followed by deposition of a $1.5 \mu$ m-thick AlN layer. Then, the PnC devices are patterned. Subsequently, top electrodes with $100 \mathrm{~nm}$ thickness are sputtered. The entire device is released from the polysilicon substrate by a $\mathrm{XeF}_{2}$ isotropic dry-etch process.

tungsten film for the contacts. The $\mathrm{W}$ film is laid on the etched region overlying the poly-Si, and CMP is utilized to create a uniform surface and remove any excess material. Next, the bottom electrode layer $(\mathrm{Ti} / \mathrm{TiN} / \mathrm{Al} / \mathrm{Cu})$ is sputterdeposited and patterned. Then, $1.5 \mu \mathrm{m}$ of $\mathrm{AlN}$ is deposited on top. Etching of the AlN is done through a patterned photoresist layer above it. The PnC-based devices are formed by pattering the AIN layer. Metal patterning of the contact is performed by first sputtering $200 / 50 / 50 \mathrm{~nm}$ of $\mathrm{Al} / \mathrm{Cu} / \mathrm{TiN}$ followed by etching. This layer will serve as the top electrode for the transducers (Fig. 2(f)). The width of each electrode depends on the targeted center frequency and the average sound velocity of the electrodes. Next, a $100 \mathrm{~nm}$ PETEOS oxide layer is deposited over the entire device. Anisotropic etching of this oxide is done to open the electrode pads and to expose the poly-Si layer by selective etching of oxide to poly-Si. The poly-Si layer is released by isotropic etching with $\mathrm{XeF}_{2}$ (Fig. $2(\mathrm{~g})$ ). This results in a suspended air-bridge device configuration, thus reducing loss to substrate. Several devices with the same PnC structure but with different electrode widths were fabricated in order to characterize the entire frequency range from $1.05 \mathrm{GHz}$ to 1.25 GHz. For each PnC device, an identical device with no inclusions (AlN slab) was also fabricated for normalization purposes.

A scanning electron microscope (SEM) image of a fabricated AIN PnC is presented in Fig. 3(a). Integrating the appropriate electrode size, acoustic waves are launched toward the PnC devices. The zoomed-in top view (Fig. 3(b)) of the unit cell is also shown. The response of the PnC devices was measured using a direct 2-port network analyzer. All measured transmission data are then normalized with reference devices, which consist of full AlN slabs with no pattern (matrix). For the cavity, the ratio of the transmission to that of the matrix was calculated. 


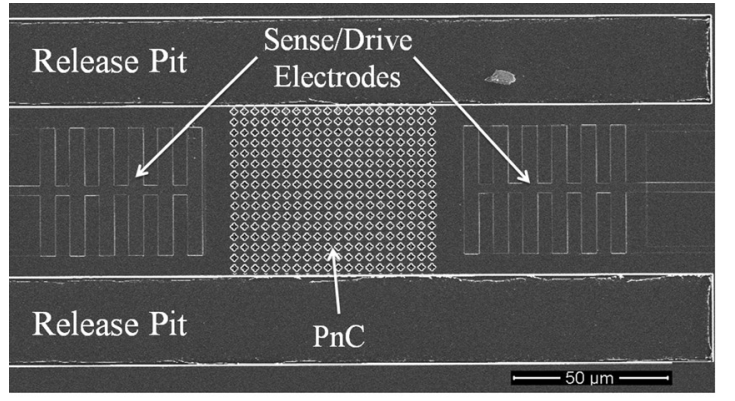

(a)

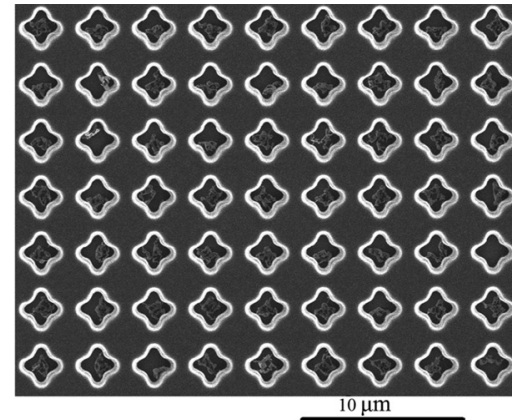

(b)
FIG. 3. (a) SEM of a fabricated AIN PnCs with drive and sense Al electrodes separated from each other by 15 layers of phononic crystals. (b) SEM figure of PnCs (top view).

\section{RESULTS AND DISCUSSION}

Transmission spectra along the $\Gamma$-X direction through the $\mathrm{PnC}$ were measured and normalized with that of the slab. The results for both the PnC and slab are presented in Fig. 4. Low transmission coefficients in the frequency range of $1110<f<$ $1175 \mathrm{MHz}$ can be observed. This is in excellent agreement with the theoretical predictions shown in Fig. 1(a). A bandgap over this frequency range allows for the creation of other types of devices necessary for RF communications. In addition to the PnCs, a microresonator was also simulated and realized experimentally. The FEM results are in agreement with experimental results; however, FEM shows a deeper bandgap as expected. This is due to losses in the experiment such as substrate losses, ${ }^{24,25}$ material losses, ${ }^{5}$ insertion losses,${ }^{26}$ and due to the Interdigital Transducers (IDTs) only detecting the longitudinal and flexural waves. ${ }^{27}$ These experimental losses cause a reduction in the transmission peaks of the devices such that when the PnC is normalized to the slab, the depth of the bandgap is shallower than modeled. The difference between experimental and numerical transmissions (See Fig. 4) is due to the fact that the FEM simulates a perfectly periodic and infinite $\mathrm{PnC}$ while the experiment features only a limited space with imperfection in the borders. This inevitable imperfection in the experiment results in its wider and deeper bandgap compared with the simulation.

Moreover, a PnC cavity was designed with the same geometrical parameters and by removing two periods of unit cells in the PnC array (W-2 cavity) and leaving three rows

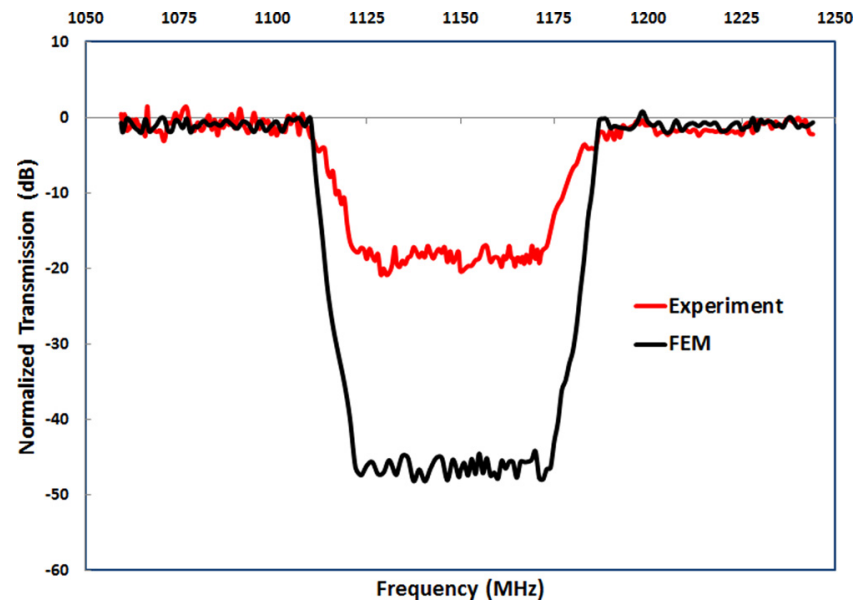

FIG. 4. Experimental (red) and numerical (black) transmission response of PnCs that have been normalized to slab (unpatterned) devices. on each side of this line defect to support certain vibrational modes across the PnC. The details of this analysis can be found elsewhere. ${ }^{28}$ Simulations also show that by removing two rows of PnCs and creating a W-2 cavity, there is one transverse mode at $1119 \mathrm{MHz}$ (out-of-plane displacement) inside the bandgap. This frequency is within the bandgap of the $\mathrm{PnC}$ and therefore allows for the confinement of energy.

The micro-cavity was also fabricated and tested using the aforementioned processing and experimental procedure. An SEM image of the fabricated devices is shown in Fig. 5. This device comprises a $6.82 \mu \mathrm{m}$ cavity enclosed by 3 phononic crystal periods on either side. The same design of the $\mathrm{Al}$ couplers and AlN piezoelectric material is used for testing the transmission spectra of the W-2 micro-cavity. The normalized transmission profile at frequencies around the resonance of this cavity was tested and is reported in Fig. 6 . As predicted, the peak associated with the flexural resonant mode of the cavity (see Fig. 6) appears in the transmission spectrum of the waves passing through the PnC structure. This peak in the transmission profile is centered at $1117 \mathrm{MHz}$, which is only $0.3 \%$ off from the resonance frequency of $1119 \mathrm{MHz}$ targeted in the simulations. The resonant peak of this micro-cavity corresponds to a quality factor per volume of about $7.6 \times 10^{17} / \mathrm{m}^{3}$. For this type of $\mathrm{PnC}$ resonator operating at atmospheric pressure, this is the

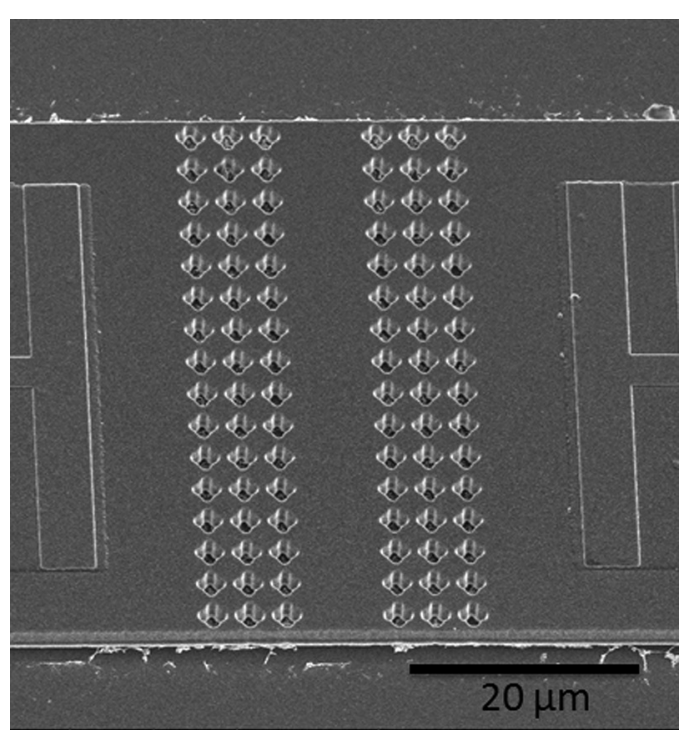

FIG. 5. SEM image of fabricated AIN resonators operating at GHz frequencies (top view). 


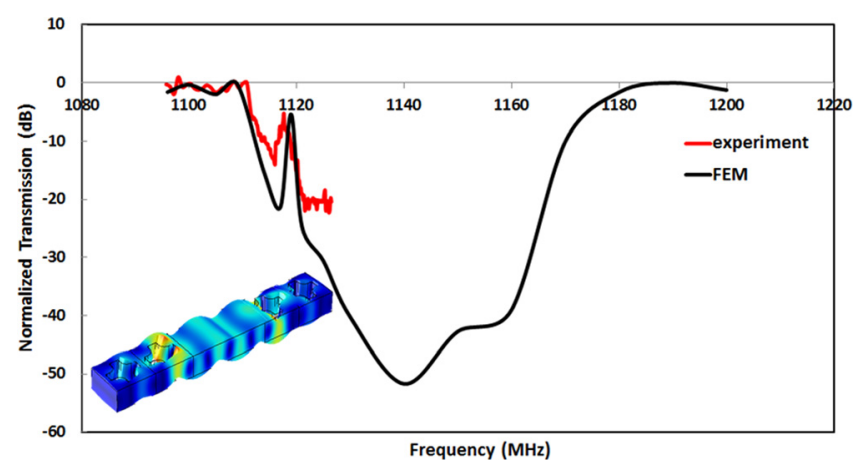

FIG. 6. Experimental and numerical results of W-2 AlN cavity. (Inset) Mode shape of the resonant frequency of the cavity structure at $1119 \mathrm{GHz}$.

highest frequency and largest $\mathrm{Q} /$ volume reported to date. The total displacement field for the confined mode in the simulations is shown in the inset of Figure 6.

Peak transmission for the W-2 cavity is limited by substrate, material, and insertion losses as previously mentioned. However, the reported Q/volume of this AlN resonator is still the highest reported to date. The $\mathrm{Q} /$ volume can be further increased by minimizing the losses previously mentioned, operating the devices in a vacuum to minimize losses to the surrounding atmosphere, and by surrounding the W-2 cavity with more periods of the $\mathrm{PnC}$, thereby increasing confinement.

\section{CONCLUSION}

Phononic crystals with a square lattice of cross-shaped air inclusions in a matrix of AlN films were realized. The devices featured an acoustic bandgap ranging from 1110 to $1175 \mathrm{MHz}$. A W-2 cavity operating in this range of frequencies was also fabricated and investigated. The cavity utilized three periods of PnCs in each side, and the experimental results evidenced a quality factor of $7.6 \times 10^{17} / \mathrm{m}^{3}$ which is the highest $\mathrm{Q} /$ volume reported to date. The realization of AlN PnCs and microcavities functional at ultra-high frequencies paves the way for developing new technologies to shrink the devices used in the RF communications. This microresonator is applicable for a new generation of filters for $\mathrm{RF}$ communications with smaller dimensions.

\section{ACKNOWLEDGMENTS}

M.G.B., S.A. and Z.C.L. acknowledge support from the National Science Foundation Division of CMMI under Award 1056077. Portions of the work were carried out in UNM's NanoFab Facility. The work was supported by Sandia National Laboratories, a multi-program laboratory managed and operated by Sandia Corporation, and a wholly owned subsidiary of Lockheed Martin Corporation for the U.S. Department of Energy's National Nuclear Security Administration under contract DE-AC0494AL85000.

${ }^{1}$ J. O. Vasseur, B. Djafarirouhani, L. Dobrzynski, M. S. Kushwaha, and P. Halevi, "Complete acoustic band-gaps in periodic fiber-reinforced composite-materials - the carbon-epoxy composite and some metallic systems," J. Phys.: Condens. Matter 6, 8759-8770 (1994).
${ }^{2}$ M. M. Sigalas and N. Garcia, "Theoretical study of three dimensional elastic band gaps with the finite-difference time-domain method," J. Appl. Phys. 87, 3122-3125 (2000).

${ }^{3}$ A. Khelif, A. Choujaa, S. Benchabane, B. Djafari-Rouhani, and V. Laude, "Guiding and bending of acoustic waves in highly confined phononic crystal waveguides," Appl. Phys. Lett. 84, 4400-4402 (2004).

${ }^{4}$ J. O. Vasseur, P. A. Deymier, B. Djafari-Rouhani, Y. Pennec, and A. C. Hladky-Hennion, "Absolute forbidden bands and waveguiding in twodimensional phononic crystal plates," Phys. Rev. B 77, 085415 (2008).

${ }^{5} \mathrm{D}$. Goettler et al., "Realizing the frequency quality factor product limit in silicon via compact phononic crystal resonators," J. Appl. Phys. 108, 084505 (2010).

${ }^{6}$ R. H. Olsson, I. F. El-Kady, M. F. Su, M. R. Tuck, and J. G. Fleming, "Microfabricated VHF acoustic crystals and waveguides," Sens. Actuators, A 145, 87-93 (2008).

${ }^{7}$ Y. M. Soliman et al., "Phononic crystals operating in the gigahertz range with extremely wide band gaps," Appl. Phys. Lett. 97, 193502 (2010).

${ }^{8}$ M. F. Su, R. H. Olsson, Z. C. Leseman, and I. El-Kady, "Realization of a phononic crystal operating at gigahertz frequencies," Appl. Phys. Lett. 96, 053111 (2010).

${ }^{9}$ J. Chan, M. Eichenfield, R. Camacho, and O. Painter, "Optical and mechanical design of a "zipper" photonic crystal optomechanical cavity," Opt. Express 17, 3802-3817 (2009).

${ }^{10}$ M. Ziaei-Moayyed, M. F. Su, C. Reinke, I. F. El-Kady, and R. H. Olsson, "Silicon carbide phononic crystal cavities for micromechanical resonators," in 2011 IEEE 24th International Conference on Micro Electro Mechanical Systems (MEMS) (2011), pp. 1377-1381.

${ }^{11}$ S. Mohammadi, A. A. Eftekhar, A. Khelif, W. D. Hunt, and A. Adibi, "Evidence of large high frequency complete phononic band gaps in silicon phononic crystal plates," Appl. Phys. Lett. 92, 221905 (2008).

${ }^{12}$ C. M. Reinke, M. F. Su, R. H. Olsson, and I. El-Kady, "Realization of optimal bandgaps in solid-solid, solid-air, and hybrid solid-air-solid phononic crystal slabs," Appl. Phys. Lett. 98, 061912 (2011).

${ }^{13}$ N.-K. Kuo and G. Piazza, "Ultra high frequency air/aluminum nitride fractal phononic crystals," 2011 Joint Conference of the IEEE International Frequency Control and the European Frequency and Time Forum (FCS) (2011), pp. 359-362.

${ }^{14}$ A. H. Safavi-Naeini and O. Painter, "Design of optomechanical cavities and waveguides on a simultaneous bandgap phononic-photonic crystal slab," Opt. Express 18, 14926-14943 (2010).

${ }^{15}$ N. K. Kuo, C. J. Zuo, and G. Piazza, "Microscale inverse acoustic band gap structure in aluminum nitride," Appl. Phys. Lett. 95, 093501 (2009).

${ }^{16} \mathrm{M}$. Eichenfield, R. Camacho, J. Chan, K. J. Vahala, and O. Painter, "A picogram- and nanometre-scale photonic-crystal optomechanical cavity," Nature 459, 550-555 (2009).

${ }^{17}$ N. K. Kuo and G. Piazza, "Evidence of acoustic wave focusing in a microscale $630 \mathrm{MHz}$ aluminum nitride phononic crystal waveguide," in 2010 IEEE International Frequency Control Symposium (FCS) (2010), pp. $530-533$.

${ }^{18} \mathrm{M}$. Gorisse et al., "Observation of band gaps in the gigahertz range and deaf bands in a hypersonic aluminum nitride phononic crystal slab," Appl. Phys. Lett. 98, 234103 (2011).

${ }^{19}$ A. Tazzoli et al., "A $586 \mathrm{MHz}$ microcontroller compensated MEMS oscillator based on ovenized aluminum nitride contour-mode resonators," in 2012 IEEE International Ultrasonics Symposium (2012), pp. $1055-1058$.

${ }^{20}$ N. K. Kuo and G. Piazza, "Ultra high frequency resonant cavity in aluminum nitride phononic crystals," in 2012 IEEE International Ultrasonics Symposium (2012), pp. 1236-1239.

${ }^{21}$ N. K. Kuo and G. Piazza, "X-shaped phononic crystals for side lobe reduction in ultra high frequency slanted finger inter-digitated aluminum nitride lamb wave transducers," in 2012 IEEE International Ultrasonics Symposium (2012), pp. 1145-1148.

${ }^{22}$ T. Gorishnyy, C. K. Ullal, M. Maldovan, G. Fytas, and E. L. Thomas, "Hypersonic phononic crystals," Phys. Rev. Lett. 94(11), 115501 (2005).

${ }^{23}$ P. Langlet, A.-C. Hladky-Hennion, and J.-N. Decarpigny, "Analysis of the propagation of plane acoustic waves in passive peropdic materials using the finite elemnt method," J. Acoust. Soc. Am. 98, 2792 (1995).

${ }^{24}$ S. A. Chandorkar, M. Agarwal, R. Melamud, R. N. Candler, K. E. Goodson, and T. W. Kenny, "Limits of quality factor in bulk-mode," in 21 st IEEE International Conference on Micro Electro Mechanical Systems (2008), pp. 74-77.

${ }^{25}$ S. Alaie, M. Hossein-Zadeh, M. Ghasemi Baboly, M. Zamani, and Z. C. Leseman, "Enhancing mechanical quality factors of micro-toroidal 

optomechanical resonators using phononic crystals," J.
Microelectromechanical Syst. 25(2), 311-319 (2016).

${ }^{26}$ L. Khine, M. Palaniapan, and W.-K. Wong, "6 MHz bulk-mode resonator with Q values exceeding one million," in International SolidState Sensors, Actuators and Microsystems Conference (2007), pp. 2445-2448.
${ }^{27}$ S. Alaie, M. F. Su, D. F. Goettler, I. El-Kady, and Z. Leseman, "Effects of flexural and extensional excitation modes on the transmission spectrum of phononic crystals operating at gigahertz frequencies," J. Appl. Phys. 113(10), 103513 (2013).

${ }^{28}$ M. G. Baboly et al., "The effect of stiffness and mass on coupled oscillations in a phononic crystal," AIP Adv. 3, 112121 (2013). 